\title{
Streptococcus pyogenes Antigen Measurement
}

National Cancer Institute

\section{Source}

National Cancer Institute. Streptococcus pyogenes Antigen Measurement. NCI

Thesaurus. Code C154814.

The determination of the Streptococcus pyogenes antigen present in a sample. 